\title{
Evaluation of isotopic composition of fast reactor core in closed nuclear fuel cycle
}

\author{
Georgy Tikhomirov ${ }^{1}$, Mikhail Ternovykh 1,a, Ivan Saldikov ${ }^{1}$, Peter Fomichenko ${ }^{2}$, and Alexander Gerasimov ${ }^{3}$ \\ ${ }^{1}$ National Research Nuclear University MEPhI (Moscow Engineering Physics Institute), Department of Theoretical and Experimental \\ Physics of Nuclear Reactors, 31, Kashirskoye shosse, 115409, Moscow, Russia \\ ${ }^{2}$ National Research Centre "Kurchatov Institute", Kurchatov Nuclear Technology Complex, Division of Fast and High Temperature \\ Reactors, 1, Kurchatov sq, Moscow, 123183, Russia \\ 3 Institute for Theoretical and Experimental Physics, Department of safe nuclear reactors, 25, Bolshaya Cheremushkinskaya ul., 117218, \\ Moscow, Russia
}

\begin{abstract}
The strategy of the development of nuclear power in Russia provides for use of fast power reactors in closed nuclear fuel cycle. The PRORYV (i.e. «Breakthrough» in Russian) project is currently under development. Within the framework of this project, fast reactors BN-1200 and BREST-OD-300 should be built to, inter alia, demonstrate possibility of the closed nuclear fuel cycle technologies with plutonium as a main source of energy. Russia has a large inventory of plutonium which was accumulated in the result of reprocessing of spent fuel of thermal power reactors and conversion of nuclear weapons. This kind of plutonium will be used for development of initial fuel assemblies for fast reactors. The closed nuclear fuel cycle concept of the PRORYV assumes self-supplied mode of operation with fuel regeneration by neutron capture reaction in non-enriched uranium, which is used as a raw material. Operating modes of reactors and its characteristics should be chosen so as to provide the self-sufficient mode by using of fissile isotopes while refueling by depleted uranium and to support this state during the entire period of reactor operation. Thus, the actual issue is modeling fuel handling processes. To solve these problems, the code REPRORYV (Recycle for PRORYV) has been developed. It simulates nuclide streams in non-reactor stages of the closed fuel cycle. At the same time various verified codes can be used to evaluate in-core characteristics of a reactor. By using this approach various options for nuclide streams and assess the impact of different plutonium content in the fuel, fuel processing conditions, losses during fuel processing, as well as the impact of initial uncertainties on neutron-physical characteristics of reactor are considered in this study.
\end{abstract}

\section{Introduction}

The strategy of the development of nuclear power in Russia provides for use of fast power reactors in closed nuclear fuel cycle[1,2,3]. The PRORYV (i.e. Breakthrough in Russian) project is currently under development. Within the framework of this project, fast reactors BN-1200 and BREST-OD-300 should be built to, inter alia, demonstrate possibility of the closed nuclear fuel cycle technologies with plutonium as a main source of power. Russia has a large inventory of plutonium which was accumulated in the result of reprocessing of spent fuel of thermal power reactors and conversion of nuclear weapons. This kind of plutonium will be used for development of initial fuel assemblies for fast reactors. The closed nuclear fuel cycle concept of the PRORYV assumes self-supplied mode of the reactors and regeneration by capture reaction in non-enriched uranium, which is used as a raw material.

\footnotetext{
a Corresponding author: myternovykh@mephi.ru
}

\section{Program code for simulating nuclide streams in fast reactors fuel cycle}

Existing software codes for simulation of mass transfer in open and closed nuclear fuel cycles such as MEEMS, DANESS, ICECAT, NFCSim [4], REFCO83 [5] etc. usually consider a simplified scheme of stages of change in the composition of nuclear materials. Mathematical models of changes of the isotopic composition of fuel in an infinite cell can be considered as nuclear reactors in these programs. A reactor is often represented as a simple object with user-defined value of the energy release. Multi-group approximation calculation is used in some cases. However these programs do not always take into account the features of refueling in the reactor and the irradiation history of the fuel assemblies inside the core. This approach is justified frequently due to the fact that it does not require long calculation time to obtain the needed result. Such software in some cases consider the fuel cycle of a whole country as an object for analysis. Therefore, very detailed calculations are not necessarily, 
and such evaluations are sufficient to make recommendations for the further development of the energy complex of the country. Hence, simplifications underlying in these programs correspond to this kind of tasks. Codes COSI, DESAE [6], REBUS3/VARIANT8.0 [7] can also be considered as the codes of similar type.

The code REPRORYV (REcycle for PRORYV), which simulates the processes of loading, processing, and handling of fuel assemblies in the cores of BN-600, BN$800, \mathrm{BN}-1200[8,9,10]$ and other advanced fast reactors was developed to support the PRORYV project. This code can be used to analyze closed nuclear fuel cycles. It allows to study the possibility of the self-sufficient mode of operation in fast reactor. Similar work on the modeling of neutron-physical characteristics of the stationary states of the reactor core with detailed scheme of refueling of fuel assemblies was performed for the $\mathrm{BN}-600$ reactor and was presented in [11]. A third-party software tool is used to calculate fuel irradiation and depletion on each step of fuel burnup. We can use these data to analyze external fuel cycle stages. Any code, which meets the requirements to reach the needed functionality, can be used as the third-party code.

Thus, REPRORYV is composed on modularity principle. In order to find the neutron-physical characteristics of a reactor, user can apply either precise methods (Monte-Carlo) or design methods (in this case diffusion approximation). Codes based on diffusion approximation run faster than those based on precise methods. This allows to evaluate quickly various input parameters of closed fuel cycle. After that more precise calculations of the best options can be made.

Russian code JARFR [12] is applied at present in the REPRORYV. The code is developed for evaluation of neutron-physical characteristics of fast reactors in the multi-group diffusion approximation. It is released with cross sections support based on the of, BNAB 93.01 nuclear data library [13]. The current version of the code JARFR is verified and used to study the characteristics of the project of Russian fast neutron reactors with sodium coolant, and with a variety of fuels - the current BN-600, introduced into the operation of the $\mathrm{BN}-800$ and project of BN-1200.

JARFR can calculate full-scale reactor models with separate fuel assemblies. This program enables to set various properties of different fuel assemblies in the core of the considered reactor. Thus, the program JARFR allows to take into account the history of irradiation of fuel assemblies, their unique isotopic composition at any given moment of fuel lifetime. This is the main feature that distinguishes code REPRORYV from the programs mentioned above used for simulating mass transfer in closed nuclear fuel cycle.

Various schemes of formation of composition for nitride fuel (Pu-U-N) to be loaded into a fast reactor with are discussed in this paper. This type of fuel is planned to be used in BN-1200 reactor. The influence of plutonium relative to uranium fraction on the final result is evaluated. The breeding ratio and the amount of plutonium in the reactor during 60 years of operation are also calculated.
Special input file of JARFR code is required for correct operation of REPRORYV code. Results are calculated by JARFR code on each step based on this input file. To analyze the closed nuclear fuel cycle it is needed to specify some input parameters in REPRORYV input file: cooling time, reprocessing time, fuel reload layout, distribution of isotopes into different groups, and conditions of processing for each group.

Fuel reload layout indicates an order in which fuel assemblies need to be unloaded and replaced (Figure 1). The user can specify the part of the core which is unloaded each year. Detailed example of fuel reload layout is considered below.

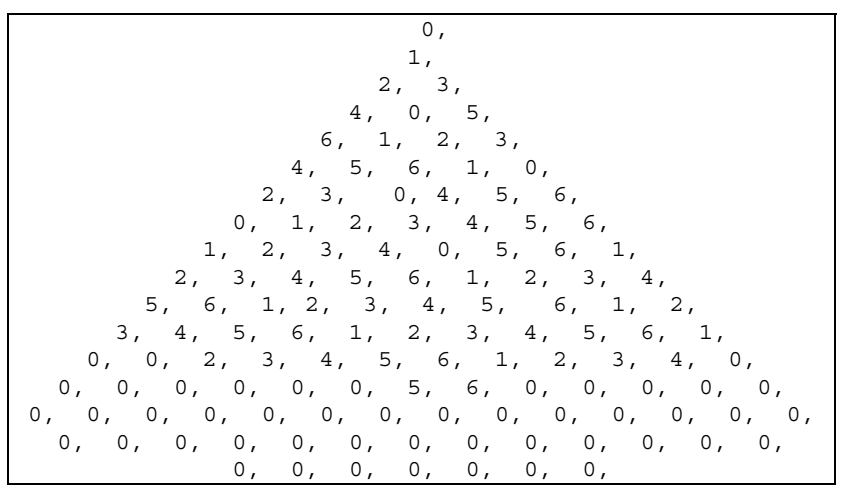

Figure 1. Fuel reload layout (60 degrees symmetry sector).

It is important to separate isotopes correctly to the groups and then operate with groups of isotopes on the stages of reprocessing. User may choose one of three options for each group as follows:

1. Do not change group concentrations, i.e. retain the mass of group as it was unloaded;

2. Remove the group after recycling (assign concentrations to zero);

3. Assign the initial value of the mass of the group, i.e. add or remove mass of the group so that the mass would be the same as at the first loading. When this option is selected, concentrations of each isotope can be changed while the total mass should be the same as at the initial step.

Thus, the user can allocate separate groups of plutonium isotopes (to change mass to its original value, "removing the excess of plutonium"), uranium (to add mass to the initial level by adding ${ }^{238} \mathrm{U}$ ), structure materials (to leave them as they were) and actinides (remove them from reprocessed fuel, or leave them in case of the post-combustion scenario).

Various reload layouts can be introduced according to designer specifications. The effect of plutonium losses on fuel reprocessing stages is studied. Small loss of plutonium is allowable under equipment pollution in processes of separation of fuel fractions and fission products. To specify the input data of REPRORYV, the data from publications on the isotopic composition of fuel, energy release in the core composition and structural materials are used in this work $[14,15]$.

\section{Uncertainties and errors of calculations}


Advantage of the code REPRORYV is the possibility of direct calculation of the errors in the concentrations on the basis of errors in the input data. For this purpose, third-party code of nuclide kinetics BPSD (Burning and Poison calculation System with Deviation) [16] is used in REPRORYV code. The BPSD code was developed in the Institute of the Problems of the Development of Nuclear Power of the Russian Academy of Sciences (IBRAE RAS). This code allows to calculate any chains of nuclide transitions both for actinides and fission products. Library RUSFOND (IPPE) simulating up to about 1500 fission products and up to 40 actinides is used as database for BPSD code. Feature of the program is the direct calculation of the above-described errors: errors of the used numerical methods and errors of the input data, which are set inside the program. The result containing numerical values of the errors allows the user to take into account in their work the possible inaccuracies associated with the simulation, to get the confidence interval, the maximum deviation value, etc.

The user of the BPSD code has to assign the input data for the beginning of every step of burnup that is shown in figure 2 . These input data are:

- Concentrations of actinides.

- Multi-group (26 groups) cross sections of fission, radioactive capture, $(n, 2 n)$ and $(n, 3 n)$ reactions.

- Neutrons spectrum (26 groups).

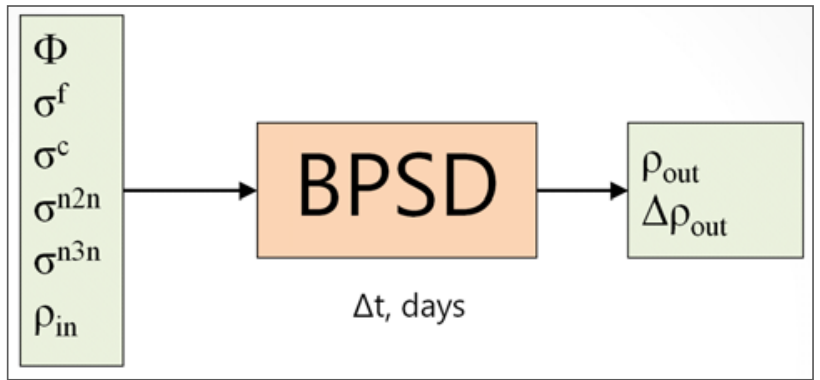

Figure 2. Simplified scheme of the BPSD program with the output of errors of the concentrations

At the moment the application of the BPSD code to estimate the uncertainty of the concentrations for the end of reactor operation, is under testing. This module will allow to include function of estimation of uncertainties of the mass for any time moment of reactor operation into the REPRORYV code. These uncertainties will allow to estimate the error of the resulting functionals, such as reactivity and density effects of reactivity.

\section{Processing}

Simulation of the recycling process in the REPRORYV code is based on the assumption that the discharged spent fuel should consist of two fractions: the product and the waste Scheme of processing in REPRORYV code taking into account uncertainties in isotopic composition is shown in figure 3 .

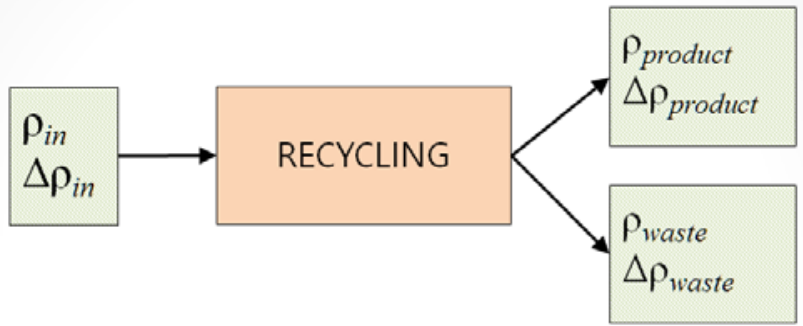

Figure 3. Scheme of processing in REPRORYV code taking into account uncertainties in isotopic composition.

In general case, the schema of processing is nonlinear and depends on many factors. Some of the software codes specifically designed to simulate the process of fuel reprocessing, separation of individual sections of processing lines, calculation of the processing coefficients relying on the chemical reactions, refining, etc. In the REPRORYV code, all processing steps are replaced by one common stage, after which it is already clear what part of the isotopes is the product and what part of the isotopes is waste. The general scheme of closed nuclear fuel cycle simulated in the task by the code REPRORYV is shown in figure 4.

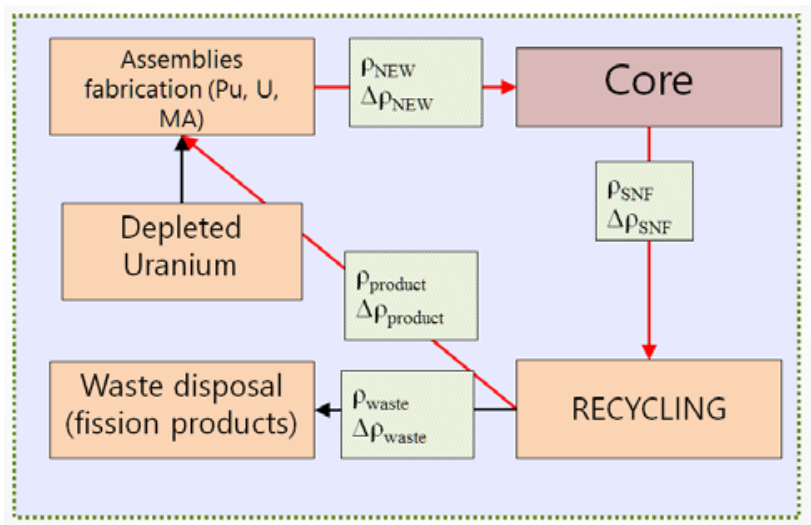

Figure 4. The general scheme of closed nuclear fuel cycle simulated in the task by the code REPRORYV

This simplified scheme allows us to specify vector of coefficients of processing for each isotope, removing the complexity of the scheme of spent fuel reprocessing. After processing the waste is directed to the burial, and the product is used to create new fuel loading for the reactor. Vector of coefficients of the separation of the product from the waste for the major isotopes $(\sim 20-30$ isotopes) is assigned as the parameters of the separation process. Values of these coefficients of the separation were obtained with account of losses and errors. 


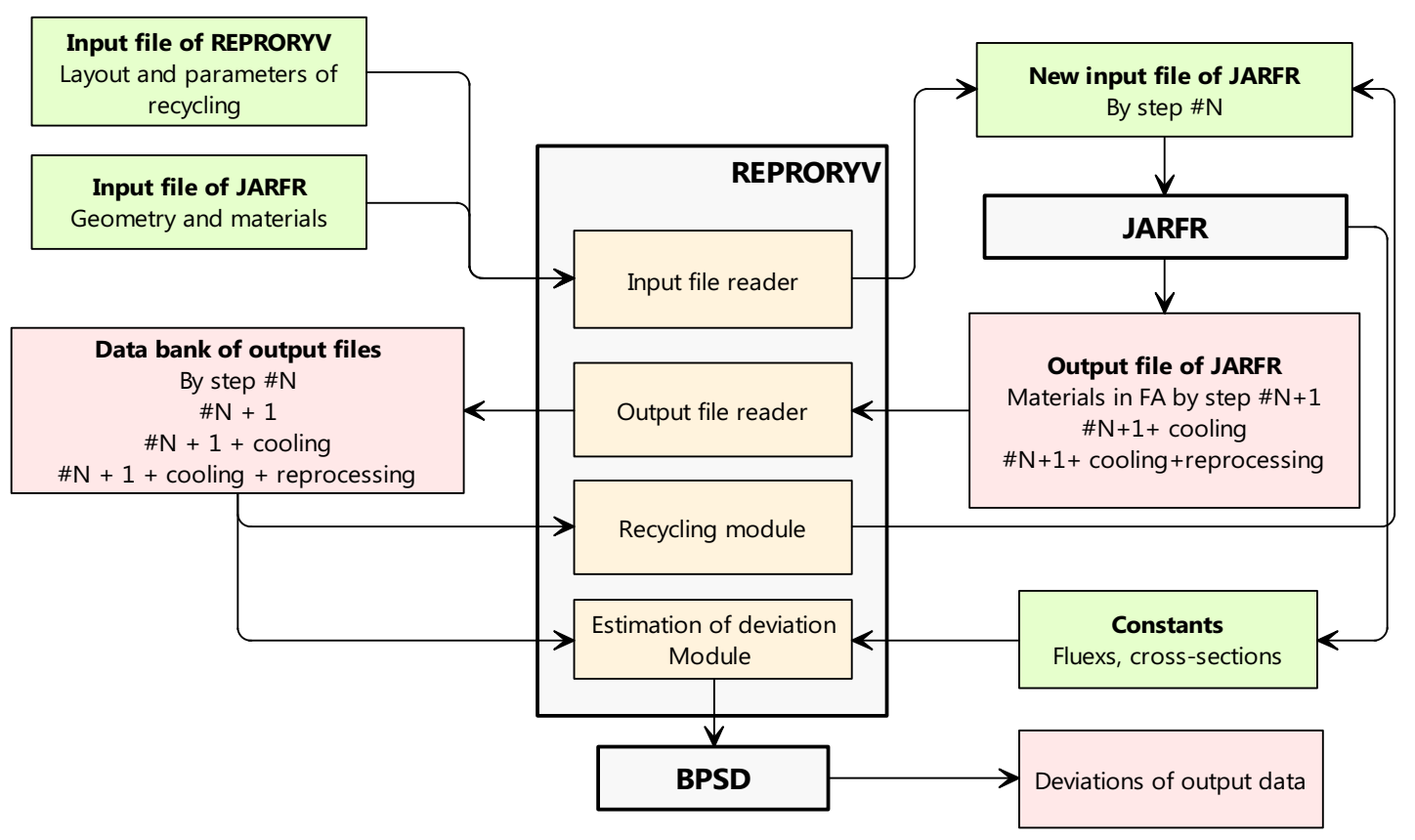

Figure 5. The general scheme of operation of the program REPRORYV

The following equations are used at each step of processing to obtain the new concentration values:

$$
\begin{gathered}
\vec{\rho}_{\text {product }}=\vec{k}_{\text {recl }} \times \vec{\rho}_{\text {in }} \\
\vec{\rho}_{\text {waste }}=\vec{k}_{\text {waste }} \times \vec{\rho}_{\text {in }} \\
\vec{k}_{\text {recl }}+\vec{k}_{\text {waste }}+\vec{d}=\overrightarrow{1} \\
|\vec{a}|>0 \\
|\vec{b}|>0 \\
|\vec{d}|>0
\end{gathered}
$$

where $\vec{\rho}_{\text {in }}$ is vector of concentrations of isotopes at the beginning of the procedure of processing;

$\vec{\rho}_{\text {product }}$ is vector of concentrations of isotopes in the product after processing;

$\vec{\rho}_{\text {waste }}$ is vector of concentrations of isotopes in the waste after processing;

$\vec{k}_{\text {recl }}$ is vector of coefficients of processing. Each component of this vector contains coefficients of processing for each of the major isotopes. Coefficient of processing means the share of the transition of each isotope in the product;

$\vec{k}_{\text {waste }}$ is vector of the coefficients of the transition to waste. Each component of this vector contains coefficients of transition to the waste for each of the major isotopes. Coefficient of transition to waste means the share of the transition of each isotope into the waste;

$\vec{d}$ is vector of the share of losses in the processing specified by the user.

It is obvious that each element of these vectors is greater than zero, and the sum of these vectors is equal to a unit vector (the law of conservation of mass).

There are certain difficulties in obtaining accurate values of losses at each operation of the processing. However, assignment of approximate values dives a possibility for the application of the algorithm with accurate data, which can be obtained experimentally directly in the processing plant.
After starting the program with all assigned required characteristics of the fresh fuel, irradiation parameters, cooling time, etc., the output file will contain the isotopic composition of the fuel for loading into a new reactor, its mass, neutron-physical characteristics of all active core and its parts, the errors of the obtained values.

The general scheme of operation of the program REPRORYV is presented in figure 5.

\section{Core reloading layout}

Reloading layout of the fuel assemblies in a BN-type reactor core was accepted as follows. Four different variants $\mathrm{T} 1-\mathrm{T} 4$ of plutonium mass content relative to uranium in the initial nitride fuel were considered. It was assumed that plutonium content was $12.6 \%$ (T1), $12.8 \%$ (T2), 13\% (T3), and 14\% (T4). Plutonium isotopic composition corresponded to that of discharged from the spent nuclear fuel of VVER-440 reactors [14,17] in all four cases. Initially fresh fuel assemblies were loaded in the core. In the first step of reloading after 330 days of operation, 12 fuel assemblies of 72 (1/6 part of the core) marked by index of "1" (first lot of fuel) in Figure 1 were unloaded and placed into intrareactor storage for cooling untill the activity of these fuel assemblies would decrease to the level when they could be reprocessed. This time of cooling was evaluated as two years in our case. New fuel assemblies with the same initial composition were placed to the positions of unloaded fuel assemblies during following 35 days. The time of operation during 330 days and time for reloading of 35 days were assumed for all steps of reloading. The total time was equal to one year. In the second step of reloadong after next 330 days of operation, fuel assemblies marked by index of "2"(second lot of fuel) were unloaded for cooling and replaced by new fuel assemblies during 35 days. Then reactor 
operated again during 330 days and so on. After two years of cooling, fuel assemblies of type "1" that have been loaded first, were sent for recycling. Several variants of reprocessing were considered. Actinides could be removed from the fuel (S1) or could be retained in the fuel (S2). In the process of separation of fission products, a small amount of plutonium could be extracted along with fission products. The amounts of plutonium as impurity to separated fission products of $0.1 \%(\mathrm{R} 1), 0.5 \%$ (R2), and $1 \%$ (R3) were considered. The time of reprocessing was assumed as one year. In the third step of reloading the fresh fuel assemblies were place to position " 3 " and the reactor operated for following 330 days. Finally in the fourth step of reloading, recycled fuel which was reprocessed over the last year was placed to position "4" (on the contrary to the fresh fuel as in the previous steps), composed of the first lot of fuel. Then, in following steps of reloading, fuel assemblies that have been unloaded and reprocessed after the second step (second lot) would be placed in position "5", after the third step - in position "6", etc. Thus, the reactor comes to steady state mode of operation, if the vector of plutonium (the fraction of each plutonium isotope in the mass of plutonium) at each following step of the refueling of the active core remains constant (self-sufficient plutonium mode)

\section{Results}

Geometry parameters and isotopic composition of the model of a BN-type reactor core were used as input for JARFR to obtain the results on multiplication factor and mass of plutonium at the end of the time interval. Fuel reloading layout and modes of recycling were specified in REPRORYV code. Results for T1, T2, T3, T4 variants were obtained using options S1 + R1. Results of different $\mathrm{S}$ and $\mathrm{R}$ variants were obtained using T3 variant. Each fuel assembly was homogenized in the active core. All results correspond to fuel density $11.5 \mathrm{~g} / \mathrm{cm}^{3}$. The reactor was set to the critical state $(\operatorname{Keff}=\sim 1)$ by changing plutonium content in the active core only in the initial period of time. The tolerance of calculation of the multiplication factor in each case was equal to $10^{-5}$. The rule "mass of the unloaded fuel assemblies should be equal to the mass of the loaded fuel assemblies in each of the cycles of refueling" was accepted for the formation of the new fuel composition by processing.

Figure 6 shows the dependence of the reactivity of the reactor core (in percent) on time (the lifetime of the reactor is 60 years) for different values of the plutonium share in the fuel in the initial moment of time. It is seen that all four types of fuel tend towards similar levels of reactivity. It should be noted that at the initial time moment the reactivity value for fuel with plutonium fraction of $14 \%$ is 3.8 times higher with respect to the value of reactivity for fuel with $12.6 \%$ of plutonium. Nevertheless we can conclude that the optimal value of a share of plutonium will be a value from $12 \%$ to $14 \%$, because reactivity change during the reactor operation will be the smallest one just for this range of plutonium share. For this reason, there will be no need in high margin of reactivity in the control rods and their frequent moving at large amplitudes in the active core. This increases the overall safety level of the reactor plant.

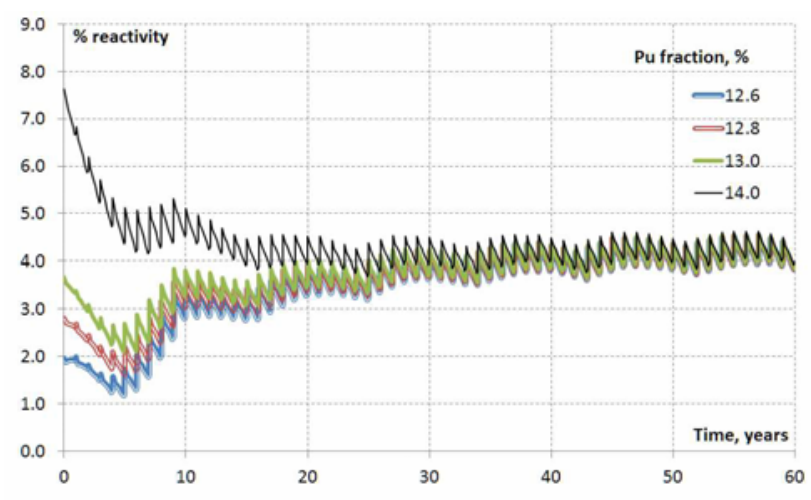

Figure 6. The dependence of reactivity of the core on time (the life cycle of the reactor is 60 years) for different values of plutonium share in fuel in the initial time

If we consider this dependence in the initial 3 years as shown in figure 7 , we can see more smooth dependence of reactivity during one cycle (one year of reactor operation between refueling) for active core with initial share of $12.6 \%$ plutonium in fuel assemblies. Thus, it is possible to assume that if we remove accumulated excess of plutonium mass from the active core of the reactor, the excess reactivity in the core will be close to zero. Thus, after 60 years the difference between three types of plutonium loads actually disappears. Changing of reactivity between reloadings is the same. This can be seen in figure 8 for the end of reactor operation.

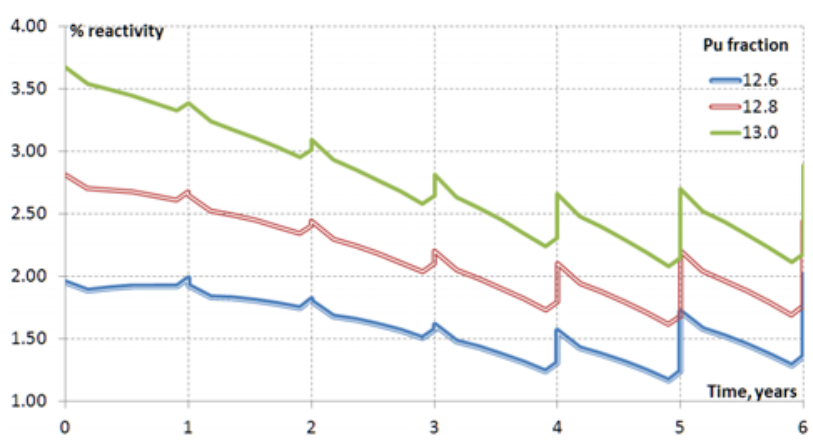

Figure 7. The dependence of reactivity of the reactor core on time in the beginning of the campaign (3 years) for different values of initial plutonium share in fuel

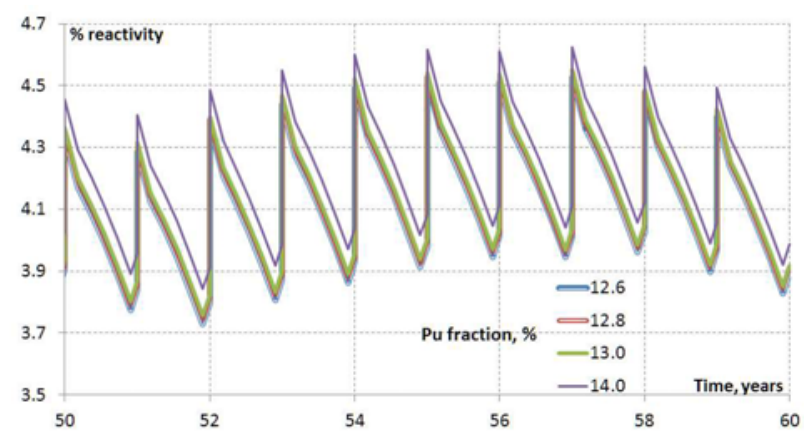


Figure 8. The dependence of reactivity of the reactor core on time at the end of reactor operation (from 50 to 60 years) for different values of initial plutonium share in fuel

Figure 9 shows the dependence of the mass of plutonium in the reactor core at the end of each microcampaign depending on time for different loads of active core containing different shares of the plutonium in the total mass of fuel. It is seen that all the four active cores tend to some equilibrium value of the rate of plutonium accumulation. At the initial moment of time the ratio of the mass of plutonium when passing from $14 \%$ to $12.6 \%$ makes 1.1106 . At the end of reactor operation this ratio is only 1.0019 .

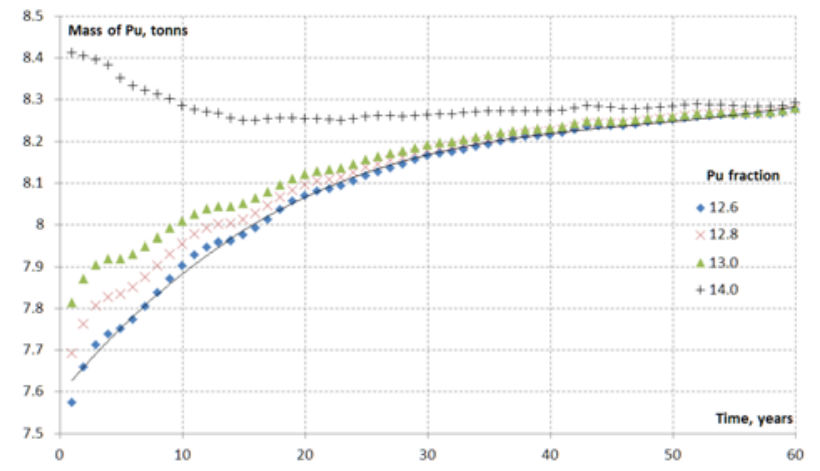

Figure 9. The dependence of the mass of plutonium in the reactor core at the end of each microcampaign on time for active cores containing different shares of plutonium in the total mass of the fuel.

Small fluctuations of plutonium mass are associated with the fact that in the first three microcampaign, fuel lots are loaded with initial content of plutonium (i.e. as it was the content at the beginning of reactor operation) instead of unloaded plutonium. Figure 10 shows the timedependence of the plutonium mass loaded in each step from the same fuel lot. Since the time of irradiation of one fuel lot in the reactor is 6 years, followed by 2-year cooling in interim storage and 1-year processing, it is obvious that this fuel lot (without fission products and minor actinides) can be loaded again every 9 years. Therefore, the neutron-physical characteristics and the masses of isotopes will decrease shortly in every 9 years due to the fact that the plutonium is not added to reactor during the first three years. The black line in figure 12 shows the trend line. It is seen that, as for reactivity, the value of the mass of plutonium loaded in each fuel lot in the reactor, goes to the equilibrium value (mass does not change as much as at the initial time).

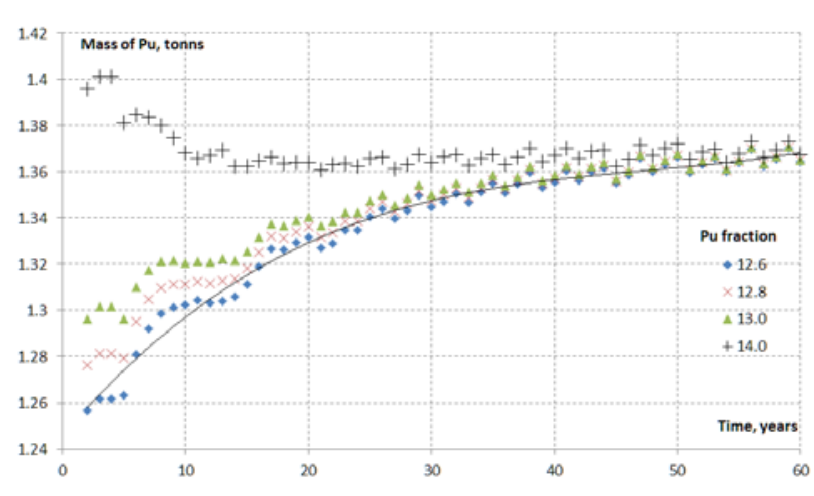

Figure 10. Dependence of plutonium mass downloaded at each step in one fuel lot on time for active cores containing different shares of plutonium in the total mass of fuel.

This rule is met also for fuel lots. In each fuel lot after 9 years it is necessary to add a smaller amount of plutonium to support the operation of the reactor (for the same mass of each fuel assembly, see table 1). From 4-th cycle the "excess" of plutonium accumulated in an amount of not more than $1 \%$.

Table 1. Change (\%) of the mass of plutonium during its production in each fuel lot during the reactor life cycle

\begin{tabular}{|r|r|r|l|r|r|r|r|}
\hline & \multicolumn{7}{|c|}{ No of campaign } \\
\hline Lot & 1 & 2 & 3 & 4 & 5 & 6 & 7 \\
\hline 1 & - & -3.8 & -2.1 & -1.3 & -0.7 & -0.5 & -0.3 \\
\hline 2 & - & -3.3 & -1.8 & -1.3 & -0.6 & -0.5 & -0.2 \\
\hline 3 & - & -3.3 & -1.9 & -1.4 & -0.6 & -0.6 & -0.2 \\
\hline 4 & - & -3.4 & -2.2 & -1.2 & -0.7 & -0.4 & -0.3 \\
\hline 5 & - & -2.4 & -1.8 & -0.9 & -0.7 & -0.3 & -0.3 \\
\hline 6 & - & -2.1 & -1.6 & -0.8 & -0.7 & -0.2 & - \\
\hline 7 & - & -2.1 & -1.3 & -0.8 & -0.5 & -0.3 & - \\
\hline 8 & - & -1.9 & -1.0 & -0.8 & -0.3 & -0.4 & - \\
\hline 9 & - & -2.1 & -1.1 & -0.8 & -0.3 & -0.4 & - \\
\hline
\end{tabular}

To comply with the law of conservation of mass of unloaded and loaded fuel assemblies, the mass of depleted uranium added during the processing was varied. Since the mass of plutonium is increased even with the account of its burning due to fission reactions, the total mass of uranium in the active core decreases with time, see figure 11. Fluctuations in the uranium mass arise in this case for the same reason as fluctuations of plutonium. 


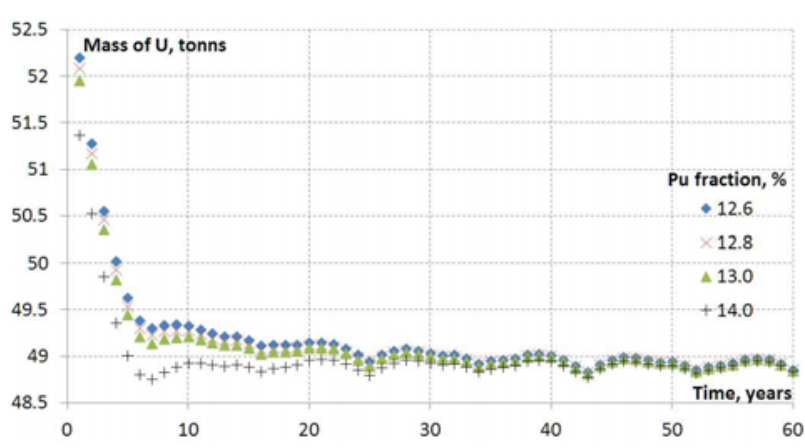

Figure 11. The dependence of uranium mass in the reactor core on time at the end of each microcampaign for active cores containing different shares of $\mathrm{Pu}$ in the total mass of the fuel.

Figure 12 presents dependence of reactivity of the reactor core on time for different values of plutonium loss during processing. In the initial time, dependence of the reactivity is the same for different values of plutonium loss. The influence of plutonium losses on neutronphysical characteristics will be maximum only at the end of reactor campaign. The ratio of the values of reactivity corresponding to loss of plutonium of $0.1 \%$ and $1.0 \%$ at the end of the reactor operation is 1.13 .

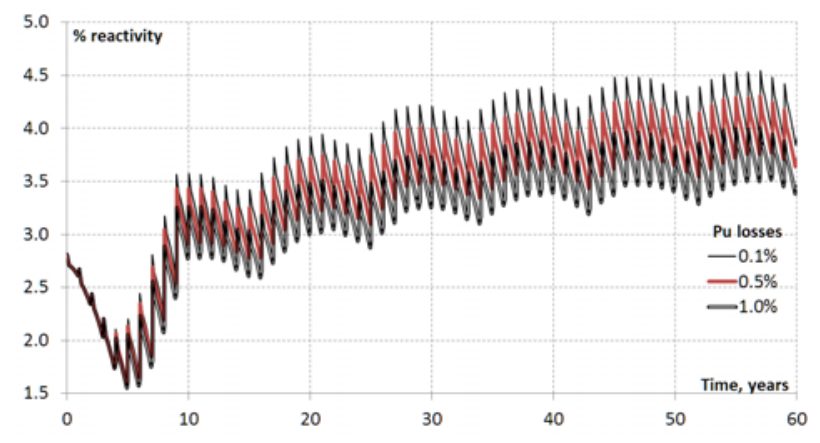

Figure 12. The dependence of reactivity of the reactor core on time (the life cycle of the reactor is 60 years) for different values of plutonium loss during processing.

Figure 13 shows the dependence of reactivity of the reactor core on time (campaign of the reactor is 60 years) when minor actinides are removed from the fuel or keep in fuel.

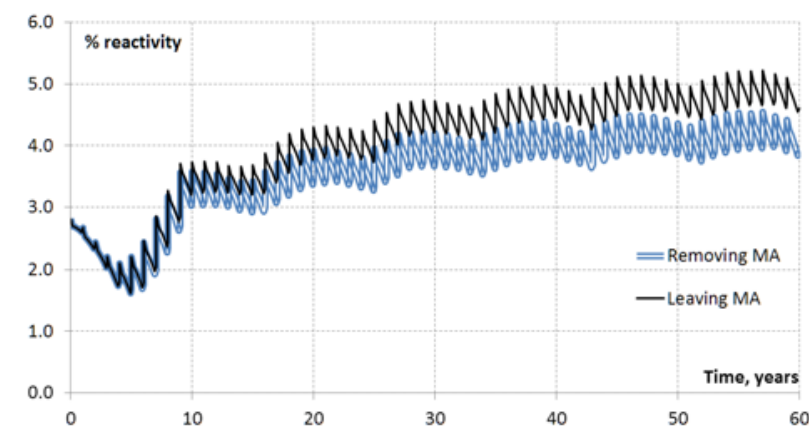

Figure 13. The dependence of reactivity of the core on time (the life cycle of the reactor is 60 years) for removal of minor actinides and leaving them in the fuel.

In the initial time, values of reactivity to these two variants coincide. For the end of reactor operation, actinides increase the reactivity compared to case of their complete removal during the processing by 1.18 times.

\section{Conclusions}

The paper deals with evaluation of fuel handling in fast reactors in the closed nuclear fuel cycle. To solve these problems, the REPRORYV program code is developed. It simulates the nuclide streams in out-of-reactor stages of the closed fuel cycle. Existed verified code JARFR is used for the calculation of neutron-physical characteristics of the core. Various options for nuclide streams are considered with a representative full-scale model of a fast reactor with sodium-cooled high-power. The changes of multiplication factor and mass of plutonium in the closed nuclear fuel cycle for a Russian BN-type fast reactor with use of the REPRORYV code are evaluated. Different scenarios of fuel reprocessing assuming the removing or retaining of actinides, taking into account various plutonium losses on reprocessing steps were considered. The questions of influence of the initial content of plutonium in nitride fuel (Pu-U-N) and the impact of the initial parameters to the possibility of fuel self-sufficient mode of the reactor during the whole period of its operation are studied.

\section{References}

1. V.P. Alferov, A.I. Radaev, M. V. Shchurovskaya, G. V. Tikhomirov, N.A. Hanan, F.A. Van Heerden, Ann. Nucl. Energy 77 (2015) 273-280.

2. A.S. Gerasimov, V.N. Kornoukhov, I.S. Sald'ikov, G.V. Tikhomirov, At. Energy 116 (2014).

3. B.R. Bergelson, V.V. Belonog, A.S. Gerasimov, G.V. Tikhomirov, At. Energy 107 (2009).

4. Physics and Safety of Transmutation Systems. A Status Report C OECD 2006 NEA No. 6090.

5. REFCO83, Nuclear Fuel Cycle Cost Economics Using Discounted Cash Flow Analysis. http://www.oecdnea.org/tools/abstract/detail/nesc1065/

6. E. Andrianova., V. Davidenko, V. Tsibul'Skii.. Atomic Energy, 105 (6), 303-306 (2008).

7. REBUS3/VARIANT8.0, Code System for Analysis of Fast Reactor Fuel Cycles. http://www.oecdnea.org/tools/abstract/detail/ccc-0653/.

8. V. Poplavsky, A. Tsiboulia, Yu. Khomyakov, V. Matveev, V. Eliseev, A. Tsikunov, B. Vasiliev, S. Belov, M. Farakshin. Core design and fuel cycle of advanced fast reactor with sodium coolant. International Conference on Fast Reactors and Related Fuel Cycles - Challenges and Opportunities - December 7-11, 2009, Kyoto, Japan.

9. B. Vasilyev, S. Shepelev, M. Ashirmetov, V. Poplavsky. BN-1200 Reactor Power Unit Design Development. International Conference on Fast Reactors and Related Fuel Cycles: Safe Technologies and Sustainable Scenarios. Paris, France, 4-7 March 2013. 
10. A. Vasiliev, P. Fomichenko, P. Alekseev, V. Nevinnitsa, S. Belov, B. Vasiliev, V. Evseev, M. Farakshin. Preprint Kurchatov Institute IAE-6275/4 (2003).

11. A. I. Nevinitsa, V. I. Matveev, V. Yu. Stogov, V. A. Cherny, Problems of Atomic Science and Engineering, Series: Physics of Nuclear Reactors, 2, 53-57 (2011).

12. L.N. Yaroslavtseva. The JAR Code System for Nuclear Reactor Neutronics Calculations. VANT, issue 8 (37), 1983, pp. 41-43.

13. G. N. Manturov, M. N. Nikolayev, A. M. Tsybulya, Problems of Atomic Science and Engineering, Series: Nuclear Constants, 1, 4-12 (1996).

14. R. Prodanova. Calculation of isotope composition of WWER- 440 spent fuel assembly by the NESSELNUKO code system on the basis of the ISTC burn up credit project data. Institute for Nuclear Research and Nuclear Energy Bulgarian Academy of Sciences

15. S. B. Belov, A. V. Kiselyov, E. V. Marova, M. R. Farakshin, M. N. Zizin, P. A. Fomichenko, K. F. Raskach, M. Yu. Semyonov, Problems of Atomic Science and Engineering, Series: Physics of Nuclear Reactors, 4, 77-82 (2014).

16. A. Belov, E. Seleznev. Atomic Energy, 108, 321324 (2010).

17. B.R. Bergelson, V.V. Belonog, A.S. Gerasimov, G.V. Tikhomirov, VVER nuclear fuel burnup with different absorbers, At. Energy. 109 (2011). doi:10.1007/s10512-011-9351-2. 